Revue internationale P.M.E.

Économie et gestion de la petite et moyenne entreprise

\title{
Le capital de risque régional français : vocation économique - vocation financière
}

\section{Pierre-Yves Touati}

Volume 12, numéro 3, 1999

URI : https://id.erudit.org/iderudit/1008660ar

DOI : https://doi.org/10.7202/1008660ar

Aller au sommaire du numéro

Éditeur(s)

Presses de l’Université du Québec

ISSN

0776-5436 (imprimé)

1918-9699 (numérique)

Découvrir la revue

Citer cette note

Touati, P.-Y. (1999). Le capital de risque régional français : vocation économique - vocation financière. Revue internationale P.M.E., 12(3), 91-106. https://doi.org/10.7202/1008660ar

\section{Résumé de l'article}

Ce travail entend analyser comment le capital de risque régional français s'est progressivement constitué en un modèle original et performant. Deux caractéristiques qui tiennent à la grande hétérogénéité des intervenants et à leur spécialisation selon différents niveaux spatiaux emboîtés.

Notre contribution présente la stratégie des différents acteurs du capital de risque régional français et propose une typologie des intervenants qui établit une distinction entre une logique financière et une logique institutionnelle. La première sous-tend l'activité d'organismes privés qui entendent tirer profit du facteur de proximité en pratiquant leur activité dans un espace circonscrit. La seconde correspond à une prise en charge croissante, par les pouvoirs publics territoriaux, des problèmes liés à la sous-capitalisation des PME de leur milieu. Même si l'offre régionale de fonds propres aux PME demeure insuffisante, avec les synergies qui se déploient entre capitaux publics et privés, un nouveau paradigme du développement régional paraît en passe de s'imposer en France.
Ce document est protégé par la loi sur le droit d'auteur. L'utilisation des services d’Érudit (y compris la reproduction) est assujettie à sa politique d'utilisation que vous pouvez consulter en ligne.

https://apropos.erudit.org/fr/usagers/politique-dutilisation/ 


\title{
Le capital de risque régional français: vocation économique - vocation financière
}

Pierre-Yves TOUATI

CRIFES-METIS, Université Paris I

\section{MOTS CLÉS}

\section{Développement régional et local - Capital de risque Financement de la création - Financement de l'innovation Collectivités territoriales}

\begin{abstract}
RÉSUMÉ
Ce travail entend analyser comment le capital de risque régional français s'est progressivement constitué en un modèle original et performant. Deux caractéristiques qui tiennent à la grande hétérogénéité des intervenants et à leur spécialisation selon différents niveaux spatiaux emboîtés.

Notre contribution présente la stratégie des différents acteurs du capital de risque régional français et propose une typologie des intervenants qui établit une distinction entre une logique financière et une logique institutionnelle. La première sous-tend l'activité d'organismes privés qui entendent tirer profit du facteur de proximité en pratiquant leur activité dans un espace circonscrit. La seconde correspond à une prise en charge croissante, par les pouvoirs publics territoriaux, des problèmes liés à la sous-capitalisation des PME de leur milieu.
\end{abstract}

Même si l'offre régionale de fonds propres aux PME demeure insuffisante, avec les synergies qui se déploient entre capitaux publics et privés, un nouveau paradigme du développement régional paraît en passe de s'imposer en France.

\section{L'AUTEUR}

Pierre-Yves Touatı possède un doctorat en économie et est ingénieur de recherche au Centre national de la recherche scientifique (CNRS) à l'Université Paris I, Panthéon, Sorbonne. Il est responsable d'un séminaire de diplôme d'études supérieures spécialisées (DESS) d'économie de l'aménagement et développement local. Ses activités de recherche portent sur le capital de risque régional, le financement de la création d'entreprises et de l'innovation des PME régionales. Il est l'auteur de deux ouvrages et de nombreux articles sur le sujet. Adresse: CRIFES-METIS, Maison des sciences économiques, 106-112, boulevard de l'Hôpital, 75647 Paris Cedex 13, France. 


\section{ABSTRACT}

This paper analyzes how French regional venture capital now holds as a performing original model. Its original features are linked with the profound heterogeneity of operators and their specialization according to different spatial levels embodied in each other.

We present the strategies of those different operators, and a typology of them, based upon a distinction between financial logic and institutional. The first one governs the activity of private organs intending to make profit from spatial amenities provided by their local insertion. The second one comes from the growing responsibility taken by local public authorities to overcome the dramatic subcapitalization of small and medium firms.

Even if the offer of equity to those firms is still missing, a new paradigm of regional development is bound to emerge in France from the interdependencies between public and private funding.

\section{RESUMEN}

El presente trabajo pretende analizar cómo el capital de riesgo regional francés se convierte progresivamente en un modelo original y eficaz. Estas dos características están vinculadas con la gran heterogeneidad de los actores y su especialización según diferentes niveles espaciales unidos entre sí.

Nuestra contribución hace referencia a la estrategia de los distintos actores del capital de riesgo regional francés y propone una tipología de ellos, fundamentada sobre una diferenciación entre una lógica financiera y una lógica institucional. La primera sostiene la actividad de los organismos privados que buscan ganancias derivadas del factor de proximidad ligado a su inserción en un espacio circunscrito. La segunda corresponde a una toma de responsabilidad creciente, por parte de los poderes públicos territoriales, con los problemas ligados con la sub-capitalización de las PyMEs.

Aunque la oferta regional de fondos propios a las PyMEs es insuficiente, un nuevo paradigma de desarrollo regional parece imponerse en Francia con las sinergias que se despliegan entre fondos públicos y privados.

\section{ZUSAMMENFASSUNG}

Diese Arbeit analysiert anhand eines herkömmlichen und anschaulichen Modells, die fortschreitende Zusammensetzung des französischen Risikokapitals. Bei den zwei Charakteristiken des Modells wird auf eine grosse Gegensätzlichkeit der Einflussfaktoren und auf deren Spezialisierung aufgrund verschiedener Niveaus geachtet.

Der Beitrag veranschaulicht die Strategie der verschiedenen Einflüsse des französischen regionalen Risikokapitals, und schlägt eine Typologisierung der Einflussfaktoren vor. Die Typologie unterscheidet zwischen der finanziellen und institutionellen Logik. Die finanzielle Logik vertritt die Aktivität von privaten Verbindungen, welche es versteht, Profit aus einem nahegelegenen gut umschriebenen Bereich zu ziehen. Die institutionelle Logik sieht sich wachsender 
Kritik seitens örtlicher politischer Macht ausgesetzt. Die Probleme sind auf die Unterkapitalisierung der kleinen und mittleren Unternehmen zu führen.

Auch wenn das regionale Angebot an Eigenkapital der kleinen und mittleren Unternehmen ungenügend ist, scheint sich mit den Synergien, welche sich zwischen öffentlichen Mitteln und privaten Mitteln entfalten, ein neues Paradigma der örtlichen Entwicklung in Frankreich aufzudrängen.

\section{Introduction}

Durant les 30 années de croissance de l'après-guerre (les «trente glorieuses »), le financement des entreprises françaises s'est largement appuyé sur une stratégie d'endettement maximal. La croissance était alors régulière et la rentabilité d'exploitation moyenne, de près de trois fois supérieure au coût réel de l'endettement. Mais au début des années 1980, la contrainte de financement des entreprises a changé radicalement de nature, car les mécanismes classiques, reposant sur la culture d'endettement, ne répondent plus aux nouveaux besoins de financement des entreprises. Les taux d'intérêt réels sont devenus largement positifs et l'endettement, qui n'est plus source de profits, grève les finances des PME. Dans le même temps, la part des investissements immatériels s'accrôit, car les entreprises engagent ou accentuent leurs efforts d'innovation. Pourtant, le système bancaire se montre extrêmement réticent à financer ces investissements en matière grise, ainsi que les jeunes entreprises à la rentabilité aléatoire et lointaine. La baisse des taux amorcée en 1995 ne change rien à ce phénomène, car les PME pâtissent d'une grande méfiance à leur égard ${ }^{1}$ :

- les banques rationnent leurs crédits pour lutter contre l'explosion des risques à laquelle elles font face depuis 1993 ;

- le coût du crédit est corrélé négativement avec la taille de l'entreprise : entre une entreprise de plus de 100 salariés et une petite entreprise, l'écart des taux peut se monter à deux points ;

- les banquiers sont réticents à financer les phases de recherche et développement : le risque est trop élevé, pour des résultats aléatoires et lointains (Lachmann, 1996);

- le capital des entreprises innovatrices est immatériel : il s'incarne dans la maîtrise d'une technologie ou de savoir-faire, facteurs qui ne présentent pas de garanties tangibles;

- les banques n'accordent que parcimonieusement des financements à des entreprises en création : à cinq ans, leur taux de mortalité est de $50 \%$.

1. Pour des analyses plus approfondies concernant les difficultés d'accès aux financements externes éprouvées par les PME, voir Jullien et Paranque (1995) et Psillaki (1995). 
La sous-capitalisation endémique des PME, les problèmes auxquels elles se heurtent pour renforcer leurs fonds propres, représentent donc, désormais, un enjeu crucial pour le devenir de l'économie française, car c'est principalement de ces PME que l'on peut attendre le redressement des courbes d'emploi. En effet, après trois décennies de concentration des appareils industriels et des licenciements massifs, les grosses entreprises ne créent plus d'emplois, tandis que les plus petites font preuve d'un dynamisme insoupçonné. Ces 15 dernières années, elles ont accru le nombre de leurs salariés et un emploi sur deux est désormais le fait d'une entreprise en création. Mais contrairement à leurs prédécesseurs, les créateurs d'aujourd'hui ne disposent pas de fortune personnelle ; $43 \%$ d'entre eux sont d'anciens employés ou ouvriers et plus de la moitié sont d'anciens chômeurs ${ }^{2}$. Rien d'étonnant donc à ce que le capital des sociétés nouvellement créées soit, dans la majorité des cas, très peu élevé 3 . Cela constitue à court terme un danger pour l'entreprise et a fortiori pour les entreprises de haute technologie. Celles-ci éprouvent, en effet, les mêmes problèmes : durée et coûts des phases de R-D difficilement maitrisables, prix élevés des premières séries, délais de commercialisation difficiles à prévoir, retours sur investissements souvent très longs, importants besoins de fonds de roulement. Leur surface financière étriquée fragilise donc les atouts que leur confèrent leur haute compétence technique et leur créativité.

Ce besoin croissant de fonds propres de la part des PME, des plus dynamiques d'entre elles en particulier, et l'inadéquation qui se manifeste entre ce besoin et la prudence des milieux bancaires ont favorisé l'émergence du capital de risque français. En effet, le capital de risque est tout à la fois une activité et un mode de financement consistant à prendre des participations minoritaires et temporaires dans des petites et moyennes entreprises non cotées en Bourse. Il répond aux nouveaux besoins des PME en ne recherchant pas l'obtention de gains rapides et prédéterminés, mais une plus-value et un rendement à terme. De plus, le capital-risqueur fournit à ses affiliés une valeur ajoutée non monétaire qui consiste à le «porter », à l'épauler dans sa gestion et sa stratégie, à établir des rapprochements avec d'autres partenaires, à l'insérer dans de divers et nombreux réseaux (Touati, 1995).

Depuis 1985, le nombre d'organismes de capital de risque (OCR) français a plus que quintuplé, il est actuellement d'environ 300 . Cependant, le marché potentiel reste très important: le déficit en fonds propres des PME françaises est estimé à 160 milliards de francs (soit plus du triple de l'encours actuel du capital de risque français !). Mais «à côté » d'une industrie nationale du capital de risque, un capital de risque régional s'affirme avec vigueur avec des encours de participations

2. INSEE, 1996.

3. En $1994,86 \%$ des créations ou reprises se sont effectuées avec des moyens financiers inférieurs à 250000 francs, $51 \%$ avec moins de 50000 francs (Commissariat Général du Plan, 1996). 
représentant près du tiers du total national. De fait, à l'instar du venture capital anglo-saxon, où le facteur de proximité joue un rôle prépondérant, l'industrie française de l'offre de fonds propres a émergé et s'est développée dans un cadre territorial bien circonscrit : les Sociétés de développement régional (SDR) sont les pionnières de cette activité et le développement du capital de risque régional est antérieur à celui du capital de risque national (concentré en région parisienne). Au fil des décennies, divers types de structures ont assuré leur ancrage dans les milieux régionaux et ont réalisé un maillage serré du territoire en matière d'offre de fonds propres.

\section{La constitution d'un capital de risque à vocation régionale}

Les premières expériences de capital de risque en France remontent à 1955 avec la création des Sociétés de développement régional. Celles-ci devaient mobiliser l'épargne régionale pour prendre des participations dans le capital d'entreprises régionales. Mais leurs échecs financiers les inciteront rapidement à se détourner de l'offre de fonds propres pour se spécialiser dans la distribution de prêts bonifiés.

Ce sont les mesures prises par l'État, dans les années 1970 et au début des années 1980, qui vont permettre l'éclosion du capital de risque français et de sa version régionale. Sur le plan national, l'Institut de développement industriel est mis en place et le statut des Sociétés financières d'innovation (SFI) est promulgué ; en contrepartie d'un régime fiscal très favorable, ces sociétés doivent investir $80 \%$ de leur capital dans des opérations d'innovation. Sur le plan régional, des incitations financières destinées à relancer l'activité fonds propres des SDR sont élaborées. Parallèlement, le statut d'Institut régional de participation (IRP) est créé. Ces sociétés privées bénéficient de la transparence fiscale; elles ont pour vocation exclusive d'apporter des fonds propres aux entreprises régionales. Une nouvelle dynamique s'amorce à partir de 1982, avec les lois de décentralisation qui autorisent les établissements publics régionaux à participer au capital d'OCR régionaux. En utilisant largement cette possibilité, les collectivités territoriales ont été amenées à définir de véritables politiques en matière de financement en fonds propres des PME. L'émergence du sentiment que la crise affecterait, de façon irréversible, les tissus économiques régionaux, fit prévaloir, auprès de nombreux décideurs régionaux, l'idée que seule une dynamique endogène pourrait favoriser une recomposition du paysage économique.

Mais derrière la réalité géographique de l'existence d'organismes à vocation spatialement circonscrite, le capital de risque régional recouvre deux logiques distinctes : une logique financière et une logique institutionnelle ou « économique ». En effet, le qualificatif d'OCR « régional » revêt deux acceptions différentes. 
Certains organismes sont régionaux en ce qu'ils pratiquent leur activité dans un espace circonscrit. Ils sont spécialisés sur le plan spatial, comme leurs concurrents le sont au regard de certains stades de développement des sociétés ou de certaines filières. Ces OCR entendent tirer profit du facteur de proximité qui constitue la clé de voûte du venture capital aux États-Unis ${ }^{4}$ :

- la familiarité des capital-risqueurs régionaux avec les hommes, les institutions et les réseaux locaux, est un atout de premier plan pour repérer les dossiers prometteurs. Un atout également pour les affiliés du capitalrisqueur qui va s'attacher à les insérer dans ces réseaux ;

- le facteur de proximité permet d'établir une véritable relation partenariale; c'est d'ailleurs l'intuitu personce qui emporte souvent la décision d'investir;

- le portage revêt un caractère souple et non institutionnel : il ne s'agit pas de consacrer $\mathrm{X}$ jours par an à tel affilié, mais d'être disponible à tout moment pour donner une heure de son temps ;

- le suivi (contrôle de la gestion, de la stratégie, du management...) est plus aisé lorsque les partenaires sont physiquement proches ;

- enfin, nombre d'entrepreneurs préfèrent traiter avec une structure régionale sans avoir à «passer par Paris ». Cette proximité peut, de plus, permettre de lever les réticences traditionnelles des entrepreneurs français à ouvrir leur capital.

Le qualificatif de régional revêt une toute autre acception s'agissant de sociétés de capital de risque dont la finalité consiste à promouvoir le développement d'un espace. L'émergence de ces structures est le fruit d'une prise de conscience, de la part de décideurs régionaux, du rôle primordial de l'offre de fonds propres aux petites entreprises dans la recomposition des tissus économiques. Les nombreuses sociétés de capital de risque, émanations des institutionnels régionaux, ont pour mission de pallier les carences des organismes à vocation financière, ceux-ci ayant tendance à se détourner du financement, hautement risqué, des très petites entreprises, de création ou de technologie, au profit d'opérations de développement ou de transmission de moyennes ou grandes PME.

4. Les opérateurs y sont concentrés dans quelques États (32\% dans le nord de la Californie, $13 \%$ dans le Massachusetts, $6 \%$ dans le Texas) et plus particulièrement à proximité des grands pôles de recherche et de connaissances fondamentales comme la Route 128 ou la Silicon Valley. 


\section{La logique financière du capital de risque régional}

\subsection{Les OCR régionaux à vocation financière}

La définition de l'aire géographique d'intervention de ces OCR est étroitement dépendante de l'importance du marché. L'espace considéré doit pouvoir engendrer un flux de dossiers permettant une sélection rigoureuse et une bonne répartition des risques. L'aire d'intervention doit receler de 200 à 400 participations potentielles, justifiant des investissements d'un montant unitaire supérieur à un seuil (deux millions de francs environ) en deçà duquel les coûts de gestion sont jugés prohibitifs. C'est pourquoi ces sociétés à vocation financière exercent leurs activités dans un espace multirégional. Ces contraintes expliquent également l'implantation de ces OCR soit dans des régions à fort potentiel technologique ou connaissant un renouveau industriel (Rhône-Alpes, Alsace, Aquitaine), soit d'industrialisation récente (OuestAtlantique).

Ces sociétés ont pour actionnaires des agents économiques privés et, à l'exception des SFI, elles sont cotées sur un marché boursier. Quand elles en sont actionnaires, les collectivités territoriales sont extrêmement minoritaires. En revanche, de nombreux groupes industriels nationaux participent à leur capital. Cela fait partie d'une stratégie de veille technologique : la qualité d'actionnaire permet de s'informer, à un coût raisonnable, des avancées scientifiques ou techniques dans divers domaines et secteurs ; elle permet en outre d'approcher de nombreuses PME dynamiques et innovantes. Mais ces groupes industriels gagnent également à évoluer dans un environnement économique entreprenant, car la déliquescence du tissu industriel et du climat social de leur milieu constitue, à terme, une menace pour leurs activités.

Par ailleurs, de grands groupes financiers internationaux sont présents au capital de l'Institut de participation de l'Ouest (IPO) et de Siparex (Sud-Est). Ils disposent ainsi d'un excellent poste d'observation de ces tissus économiques.

Les IRP ${ }^{5}$ à vocation financière, Siparex, IPO, Auxitex (Sud-Ouest) Participex (Nord-Picardie), ne financent pas d'innovation fondamentale. Mais une bonne moitié de leurs investissements sont destinés à permettre aux entreprises de mettre en œuvre des innovations de produits ou de procédés. Cependant, le risque qu'ils acceptent de courir est nettement circonscrit : ils financent le développement et la transmission de PME saines, jamais de créations ex nihilo. En outre, leur engagement unitaire est de l'ordre de 2,5 millions de francs. Néanmoins, ces sociétés sont des acteurs de premier plan du développement régional. Elles mobilisent de

5. Depuis 1987 , le régime de Société de capital de risque (SCR) est devenu la règle fiscale de la plupart des organismes de capital de risque, mais l'appellation IRP recouvre toujours une réalité économique même si elle ne correspond plus à une catégorie juridique. 
l'épargne régionale au profit d'entreprises régionales et offrent à des PME les moyens d' accéder rapidement à la dimension nationale, voire internationale. Enfin, leur bilan économique est loin d'être négligeable. À titre d'exemple, ces 20 dernières années, Siparex, a investi près de un milliard de francs dans quelque 200 entreprises, permettant le maintien ou la création d'une quinzaine de milliers d'emplois et, de 1983 à 1996, l'IPO a investi 850 millions de francs dans 380 entreprises, permettant la création ou le maintien d'environ 8000 emplois.

Les SFI régionales ont connu, quant à elles, un parcours plus heurté. CEuvrant dans un espace circonscrit, elles ne peuvent pas se spécialiser dans certaines filières technologiques et employer des équipes d'experts, car leur flux de dossiers est moins abondant que celui de leurs consœurs nationales. C'est là un grave handicap pour financer des entreprises de haute technologie, souvent en phase de création: le taux de sinistre à cinq ans des start up très innovantes avoisine les $70 \%$ ! Ce type de financement est d'ailleurs baptisé «cocktail de la mort » par la profession...

Créée en 1984, Lorraine Créativité (dont l'actionnariat était majoritairement public), faute de n'avoir pas su mettre en œuvre les compétences nécessaires à ce type d'investissements, a cessé rapidement ses activités. Innovest (Alsace, Lorraine, Franche-Comté) et Sudinnova (Sud-Est), qui sont, quant à elles, des sociétés privées, ont tenté de relever le défi en s'adossant à leurs grands actionnaires afin d'assurer un diagnostic fiable. Parmi ceux-ci, figure, dans les deux cas, Sofinnova, la plus importante SFI française. Les régionaux font apport de leur atout de proximité : repérage et suivi performants. En contrepartie, Sofinnova apporte la qualité de ses expertises et ses compétences en matière d'industrialisation et de commercialisation de technologies de pointe. Innovest a cependant été amenée à jeter l'éponge en 1996 : la SFI n'est jamais parvenue à déborder de l'Alsace, son vivier s'est donc rapidement tari et ses résultats étaient chroniquement déficitaires.

Sudinnova, qui exerce son activité dans un espace beaucoup plus riche en projets innovants, bénéficie également du soutien d'un autre actionnaire de poids : la Société lyonnaise de banque (une banque française des plus dynamiques en matière de financement d'entreprises innovatrices). En 15 ans, la SFI lyonnaise a investi dans une soixantaine d'entreprises (pour moitié en création). Mais, bien que globalement bénéficiaire, elle a essuyé 11 dépôts de bilan pour 13 sorties positives. Une preuve, s'il en est besoin, qu'il est irréaliste de se lancer dans le « cocktail de la mort » dans un espace qui ne serait pas à même de susciter quelques success stories!

\subsection{Les sociétés de développement régional}

Dans le cadre des Programmes d'action régionale de 1955, 15 SDR sont mises en place sur le territoire métropolitain. Ce sont des sociétés privées, pour la plupart, cotées en Bourse, mais qui sont dotées d'une mission d'intérêt général. À travers ces institutions d'un type nouveau, il s'agissait de promouvoir la décentralisation 
industrielle en finançant les entreprises régionales par le biais de concours à moyen et à long terme et en fonds propres. Quarante ans après leur création, le bilan des SDR est loin d'être négligeable. Même si elles ont commis des erreurs qui, conjuguées avec la récession économique, ont provoqué, en 1992, une très grave crise.

À leur actif, les SDR, bien que n'étant pas des sociétés de capital de risque, stricto sensu, puisque leurs concours en fonds propres ont représenté, au mieux, $11 \%$ de leur activité de crédit, ont joué un rôle de premier plan dans l'émergence et la composition du paysage du capital de risque régional français :

- en 1991, avec 1,14 milliard de francs d'interventions en haut de bilan, les montants investis par les SDR ont représenté $16,5 \%$ du total des investissements des capital-risqueurs nationaux ; leur encours de participations correspondait à plus de $10 \%$ de l'encours du capital de risque français. Elles sont donc alors, globalement, les premiers fournisseurs de capitaux à risque aux PME ;

- les SDR ont été seules à répondre à la demande de sociétés aux perspectives de croissance et de rentabilité souvent modestes, dont les besoins en fonds propres étaient limités (moins de un million de francs);

- de nombreuses SDR ont favorisé le démarrage et le développement d'OCR régionaux ; elles ont participé, peu ou prou, au capital de la majorité des sociétés de capital de risque régional;

- les SDR ont joué un rôle très important dans l'éclosion et le développement du capital de risque régional en ce qu'elles ont largement participé aux syndications initiées par d'autres capital-risqueurs régionaux.

Mais, en 1992, les SDR sont affectées par une crise grave. La récession économique provoque une véritable explosion des risques dans le même temps où les investissements des PME chutent de près de $40 \%$. La crise est aggravée par la course au chiffre d'affaires menée précédemment : nombre de SDR se sont lancées dans des opérations d'envergure mais hasardeuses. Or, elles ne font pas preuve de solidarité et, à quelques exceptions, elles refusent de se regrouper dans des ensembles plus vastes, capables de s'inscrire dans une dynamique européenne. Enfin, leur acquisition récente du Crédit naval se révèle catastrophique : les pertes de cette banque, trop investie dans l'immobilier, sont colossales. Nombre de SDR se retrouvent donc avec des fonds propres insuffisants pour respecter les normes prudentielles de solvabilité et doivent renoncer à leur activité de prêt. Devant la déconfiture du réseau, l'État met en œuvre une politique d'adossement sur des actionnaires de référence ${ }^{6}$; en outre, une enveloppe de 2,5 milliards de francs de

6. La Caisse des dépôts et consignations, les Caisses d'épargne, le GAN, AXA-UAP et le Crédit agricole. 
ressources leur est allouée à taux préférentiel. Mais, en 1997, cinq SDR insolvables sont en gestion extinctive ${ }^{7}$ et deux autres ont mis un terme à leurs interventions en fonds propres ${ }^{8}$.

L'expérience des SDR illustre bien les difficultés qu'il y a à conduire une stratégie censée marier lois du marché et soutien aux politiques d'aménagement du territoire. Ce constat incite de nombreux acteurs publics régionaux à créer des sociétés de capital de risque dont la mission consiste clairement à soutenir les politiques de développement régional.

\section{La logique institutionnelle du capital de risque régional}

Le peu d'inclination des OCR régionaux, à vocation financière, pour la création d'entreprises et pour les petits dossiers s'est accentué avec l'irruption, dans les années 1990, de groupes financiers nationaux, voire internationaux, sur le marché régional des fonds propres. L'écrémage opéré par ces organismes a accru la réticence des intervenants régionaux à investir dans des sociétés aux potentialités modestes et aux possibilités de sortie lointaines. La nécessité d'une prise en charge, par les pouvoirs publics régionaux, d'une part des risques, des coûts de suivi et de portage, liés à la capitalisation de ces sociétés, s'est donc progressivement imposée. D'autant plus aisément qu'aux États-Unis, dans les années 1980, l'intervention publique en matière d'offre de capitaux aux PME devient importante. En effet, près de la moitié des États sont impliqués financièrement, et de façon conséquente, dans des fonds de capital de risque généralement spécialisés dans le financement de sociétés en création ou de technologie ${ }^{9}$.

En France, l'Institut régional de développement industriel (IRDI) de MidiPyrénées a joué un véritable rôle historique. Cet IRP a été créé en 1981 à l'initiative des pouvoirs publics régionaux qui ainsi entendaient se doter d'un outil financier destiné à soutenir le développement d'une région relativement peu industrialisée eu égard, notamment, à son potentiel technologique. La société, gérée selon les critères du secteur privé, va chercher à équilibrer un volant de participations dans des entreprises à hauts risques et un volant d'investissements moins exposés. La

7. Lordex (Lorraine), Sud-Est, Picardie, Normandie et Centrest.

8. Méditerranée, Sodler (Languedoc-Roussillon).

9. Dans les années 1990, par exemple, les États du Michigan et de Pennsylvanie ont créé cinq fonds de capital d'amorçage dotés chacun de un million de dollars ; l'État du Massachusetts alloue, annuellement, deux millions de dollars au financement en fonds propres d'entreprises en création. Les États de New York, de l'Indiana ou de l'Ohio investissent plus de 10 millions de dollars par an dans des structures de capital de risque destinées aux créations d'entreprises. 
réussite de cette expérience a mis en évidence l'efficacité d'une étroite coopération entre les pouvoirs publics régionaux et un OCR : en 15 ans, l'IRDI aura investi dans 220 entreprises (dont un tiers à risques élevés), contribuant au maintien ou à la création de quelque 5000 emplois. L'IRDI a fait la démonstration que si le rapport risque / rentabilité du capital d'amorçage ou du capital-innovation est trop élevé pour être financièrement attrayant, des organismes institutionnels peuvent, quant à eux, l'assumer. Comme on peut le constater, l'actionnaire public a deux grands mérites : il ne cherche pas à rentabiliser à tout prix ses investissements et il sait se montrer patient.

Cet exemple va inciter plusieurs collectivités territoriales à créer des OCR à vocation économique; leur aire de compétence monorégionale recoupe celle des SDR et des OCR financiers. Ces organismes institutionnels, au nombre d'une dizaine à la fin des années 1980, voient le jour dans des régions malmenées par la crise ou peu industrialisées : Lorraine, Bourgogne, Picardie, Languedoc-Roussillon, Poitou-Charentes, Champagne-Ardennes, Auvergne, Franche-Comté, etc. La participation publique est en moyenne de $30 \%$.

Depuis le début des années 1990, on assiste à une nouvelle vague de création d'OCR régionaux institutionnels. Les régions concernées par ce mouvement Rhône-Alpes, Provence-Alpes-Côte d'Azur, Centre, Alsace, Midi-Pyrénées, etc. sont bien plus favorisées tant économiquement qu'en matière d'offre de fonds propres. Dans le même temps, les régions déjà pourvues d'un OCR institutionnel en créent un deuxième, voire un troisième, destiné à intervenir en complémentarité avec celui qu'elles ont mis sur pied dans les années 1980, la particularité de ces structures récentes résidant dans la spécialisation de leurs interventions. Les pouvoirs publics régionaux veulent désormais se doter d'instruments financiers destinés soit à répondre à la faiblesse de l'offre de capitaux aux sociétés en création ou innovantes, soit à accompagner un volet particulier de leur politique.

Ainsi, en Midi-Pyrénées, pour lutter contre la polarisation de l'activité économique autour de la métropole toulousaine, la Région et les huit départements mettent sur pied Midi-Pyrénées Création. Sa vocation consiste à pourvoir aux besoins en fonds propres de sociétés localisées en milieu rural. Autre exemple : la Région lorraine crée un OCR destiné au financement des PME de l'agroalimentaire. Cette filière emploie $8,5 \%$ des salariés lorrains (soit plus que la sidérurgie ou le textile), mais près de la moitié des entreprises sont dépendantes de capitaux extérieurs à la région.

D'autres OCR à vocation économique sont quelquefois issus de la croissance de micro-organismes de capital de risque local (MOCRL) [cf. infra] qui les incite à déborder le local pour inscrire leur activité dans le cadre régional. Une logique qui peut rencontrer la démarche des collectivités de la région concernée, car celles-ci, plutôt que de créer une nouvelle entité, préfèrent parfois s'appuyer sur une société locale expérimentée, en la recapitalisant et en étendant son espace d'intervention. 
Ainsi, année après année, le maillage du territoire en OCR institutionnels devient plus serré. C'est qu'avec le retrait de nombreuses SDR des marchés des fonds propres, avec la restructuration, amorcée en 1993, du capital de risque français, avec, enfin, l'arrivée aux commandes des PME d'une nouvelle génération d'entrepreneurs, à la mentalité plus capitalistique que patrimoniale, l'apport en fonds propres tend à devenir un service public délégué. Les OCR institutionnels, qui interviennent en amont de la chaîne du capital de risque, remplissent une mission d'intérêt général : leur créneau est celui des petites PME, la part de leurs investissements en création est de 1'ordre de 30 à $50 \%$, le montant moyen de leurs participations oscille entre 400000 et 1,5 million de francs.

Mais ces structures font face à des problèmes qui tiennent à leur mission. Si elles ne sont pas soumises aux mêmes obligations de résultats que les sociétés à vocation financière, elles ne doivent pas pour autant se transformer en organismes d'assistance. Leur marge de manœuvre est donc étroite. Heureusement, au fil du temps, les pouvoirs publics régionaux ont généralement bien pris la mesure de ce risque en leur accordant une grande latitude de gestion. Les OCR institutionnels peuvent donc acquérir un professionnalisme souvent reconnu : à preuve, les fréquentes collaborations et syndications avec les OCR à vocation financière.

Un autre problème majeur réside dans les difficultés de sortie qu'éprouvent ces structures. La cession d'une participation prise en phase de création ne peut s'effectuer avant cinq à 10 ans et, à ce stade d'investissement, les risques de défaillance ou de «collage» sont élevés. Mais les OCR institutionnels ne sont pas en mesure d'équilibrer leur portefeuille avec un volant significatif de participations potentiellement plus rentables et plus rapidement «sortables », car les OCR à vocation financière écrèment les marchés. À défaut donc d'être régulièrement recapitalisés, les OCR institutionnels se trouvent plus ou moins rapidement paralysés. Mais on note une réelle prise de conscience de ce problème, de la part des actionnaires publics régionaux. Indéniablement, ils dotent «leur » organisme de moyens financiers nettement plus conséquents que dans les années 1980, à la mesure des objectifs qu'ils leur assignent.

\section{Le capital de risque local}

Quels que soient les moyens dont disposent les OCR régionaux institutionnels, leurs arbitrages ne peuvent pas satisfaire tous les milieux qui constituent leur zone d'intervention. Cela incite certains acteurs à se lancer dans le capital de risque à un niveau local. Ainsi, depuis le milieu des années 1980, on assiste à une floraison de micro-organismes de capital de risque local ; en 1999, nous estimons leur nombre à près de 80 . Par-delà leur grande diversité, ils présentent des caractéristiques communes : des moyens d'intervention modestes (moins de 15 millions de francs); des investissements unitaires faibles (50000 à 200000 francs), dans des petites 
sociétés ; une zone d'intervention étroite (département, commune bassin d'emploi); une nette orientation vers les opérations de création (plus de $50 \%$ des interventions et des montants).

Dans leur majorité, les MOCRL sont des émanations de collectivités territoriales ou d'organismes consulaires. Cette démarche s'inscrit souvent dans la poursuite d'une politique locale de soutien aux créateurs ou aux petits entrepreneurs. Après avoir agi sur l'environnement immédiat de l'entreprise, les initiatives locales se préoccupent, dans un second temps, de leurs problèmes de financement. Ainsi, des MOCRL comme Var Action, Créat'iv (Ille et Vilaine) ou Cie (Côtes d'Armor), sont, originellement, des pépinières créées par des collectivités locales ou des organismes consulaires. Eurecréation ou la Société de capital-risque de l'Ain sont, originellement, des associations ayant pour objet la formation et le parrainage de porteurs de projets.

En matière de capital de risque local, la démarche de Saménar peut légitimement être considérée comme exemplaire. Ce MOCRL a été créé en 1983, dans les Alpes-de-Haute-Provence, un département agricole sans tradition industrielle. Le capital de départ est souscrit par le Conseil général, une trentaine de communes, une soixantaine de PMI locales et sept établissements bancaires. D'emblée, cette petite structure (elle dispose de moins de trois millions de francs) adopte une stratégie très professionnelle. Saménar s'implique fortement dans le montage des dossiers et participe aux négociations avec les institutions financières et les collectivités. Un suivi étroit est de règle et le MOCRL assume une véritable fonction pédagogique à l'endroit de l'entrepreneur. Il est vrai que malgré la faiblesse de ses moyens, Saménar dispose de deux atouts : toutes les forces vives locales se sont mobilisées autour de cette expérience et la Chambre de commerce de Digne a pris à sa charge le salaire de son responsable.

Saménar a fait la preuve que même sur le plan local, même (voire surtout) pour de faibles investissements unitaires, le capital de risque nécessite un réel professionnalisme, car développement économique et rentabilité financière doivent se conjuguer pour contribuer à l'émergence et à la croissance d'entreprises pérennes. En 13 ans d'activité, Saménar a contribué à la création ou au maintien de près d'un millier d'emplois en participant au capital d'une soixantaine de sociétés. Par ailleurs, ses interventions sont à l'origine d'un effet levier de quatre sur les fonds propres et de 10 sur les crédits à moyen et à long terme. Ce dernier point mérite attention, car si les montants unitaires investis par les MOCRL ne peuvent être que faibles, ces structures peuvent produire, lorsque leur capacité de validation est reconnue, des effets de levier importants qui vont très largement contribuer à une croissance financièrement saine de leurs affiliés.

D'autres MOCRL émanent de particuliers. Certains d'entre eux entendent également apporter une réponse aux problèmes posés par un milieu économiquement défavorisé. Tel est le cas d'Herrikoa créé en 1980 pour promouvoir le 
tissu économique du Pays Basque. Cette société connaît une expansion continue attestée par le succès de ses différentes campagnes de souscription: elle compte près de 4000 actionnaires privés ! Une très forte mobilisation de l'épargne de proximité qui tient essentiellement aux particularités du Pays Basque. Le contexte politique a favorisé un projet consensuel visant à doter la population d'un instrument de développement endogène. Par ailleurs, la forte prégnance communautaire du Pays Basque - où le mouvement coopératif a toujours joué un rôle très important - a contribué à la pérennité d'Herrikoa. En 17 ans d'activité, la société a investi dans une centaine d'entreprises ; elle a contribué à la création de 1800 emplois, soit $20 \%$ des créations d'emplois du Pays Basque.

Autonomie et Solidarité est une coopérative créée par appel à l'épargne de proximité dans la région Nord ; elle compte plus de 2000 actionnaires particuliers. Sa vocation consiste à capitaliser des petites sociétés qui s'engagent à signer des contrats à durée indéterminée à des personnes en grandes difficultés d'insertion (chômeurs de longue durée, handicapés, etc.). En cinq ans, Autonomie et Solidarité est intervenu dans une cinquantaine d'entreprises et a permis la création ou le maintien de 600 emplois. Les Cigales entendent, quant à elles, impulser une dynamique en faveur d'entreprises « alternatives » ou de l'économie locale. Les membres de ces clubs mettent mensuellement de 150 à 1000 francs dans une cagnotte afin de prendre des participations dans de jeunes sociétés. Au nombre de 120, les Cigales financent des projets modestes : artisanaux ou commerciaux généralement, mais peu inducteurs d'un renouveau des tissus économiques.

Notons, enfin, que quelques MOCRL, à vocation nettement moins altruiste, sont créés par des socioprofessionnels, désireux de valoriser leur savoir-faire et leur épargne. Ces entrepreneurs suivent de façon rigoureuse la gestion de leurs affiliés tout en les faisant bénéficier de leur expérience. D'autres structures encore fonctionnent comme des clubs d'affaires : elles rassemblent quelques dizaines de particuliers désireux de réaliser des opérations lucratives en intervenant, en amont de la Bourse, dans des entreprises de proximité.

\section{Conclusion}

Face aux enjeux de l'innovation et à l'impératif du renouvellement des tissus économiques, le capital de risque régional offre des réponses pertinentes :

- il favorise l'émergence et la croissance de PME qui n'ont, souvent, ni la taille critique, ni les perspectives de valorisation requises pour bénéficier d'apports en fonds propres de la part des capital-risqueurs nationaux ;

- sa valeur ajoutée non monétaire contribue à rompre l'isolement de l'entrepreneur et à favoriser l'élargissement de l'horizon stratégique d'entreprises locales, qui sont peu enclines et fort démunies pour aborder les marchés internationaux ; 
- il offre aux PME la possibilité de mettre en œuvre de véritables politiques de recherche-développement et accélère la maturation de l'innovation en réduisant les délais de commercialisation;

- moins élitistes que les OCR nationaux, les OCR régionaux favorisent la diffusion de l'innovation et le transfert de technologie dans les régions, en donnant à des PME la capacité de procéder à des investissements en procédés ou matériels innovants.

Le capital de risque régional constitue donc un accélérateur de croissance et de développement des entreprises. L'attention croissante portée par les pouvoirs publics régionaux à la problématique des fonds propres des PME de leur milieu, d'une part, les synergies qui se déploient entre capitaux publics et privés, d'autre part, permettent d'affirmer qu'un nouveau paradigme du développement régional est en passe de s'imposer en France.

Même si l'offre régionale de fonds propres reste toujours très inférieure à la demande...!

\section{Bibliographie}

ASSOCIATION FRANÇAISE DES INVESTISSEURS EN CAPITAL (1995), Livre Blanc, Paris.

CARSALADE, Y. (1987), «Épargne de proximité et développement local», Futuribles, février, p. 3-7.

COMMISSARIAT GÉNÉRAL DU PLAN (1996), Évaluation des aides à la création d'entreprise, Rapport du groupe de travail présidé par Bertrand Larrera de Morel, Paris, La Documentation française, p. 20.

GEOFFRON, P. (1991), «Le financement des PME innovatrices : l'exemple américain », Revue française de gestion, $\mathrm{n}^{0} 84$, juin-juillet-août, p. 132-135.

INSEE (1996), «Les créations d'entreprises du premier semestre 1994 », INSEE Résultats, $\mathrm{n}^{\text {os }}$ 97-98, p. 63.

JULLIEN, H. et B. PARANQUE (1995), « Financement des entreprises et évolution du système financier », Revue internationale PME, vol. 8, $\mathrm{n}^{\text {os }} 3$-4, p. 45-52.

LaChmanN, J. (1992), Le seed capital: une nouvelle forme de capital-risque, Paris, Economica.

Lachmann, J. (1996), Financer l'innovation des PME, Paris, Economica, Gestion Poche, p. 18.

MENVILLE, J. (1992), «Le capital-risque régional : un outil de décentralisation financière ?», Rencontres de l'Institut d'économie régionale de Toulouse, février, p. 4-11.

PARANQUE, B. (1994), «Fonds propres, rentabilité et efficacité chez les PMI», Revue d'économie industrielle, $\mathrm{n}^{\circ} 67$, p. 175-177.

PSILLAKI, M. (1995), « Rationnement du crédit et PME : une tentative de mise en relation », Revue internationale PME, vol. 8, $\mathrm{n}^{\mathrm{os}} 3-4$, p. 68-75. 
TouAti, P.-Y. (1989), Le capital-risque régional et local en France, Paris, Syros Alternatives.

TOUATI, P.-Y. (1993), La dynamique internationale du capital-risque régional français, Rapport effectué pour la Délégation à l'aménagement du territoire et à l'action régionale (DATAR), Paris, publié dans Cahiers du Metis-C3E, no 102, p. 4-29.

TOUATI, P.-Y. (1994), «L'internationalisation du capital-risque régional, facteur d'ouverture des milieux », Territoires d'Europe, vol. 3, CEDRE-FEDER, p. 257-260.

TOUATI, P.-Y. (1995), «La stratégie de réseaux de la société de capital-risque lyonnaise SIPAREX », Revue d'économie régionale et urbaine, $\mathrm{n}^{0} 2$, juillet.

TREMEgE, G. (1994), Les Sociétés de développement régional et le financement des PME, Rapport d'information à l'Assemblée nationale, $\mathrm{n}^{\circ} 1841$, p. 37-45.

TURPIN, M. (1996), Capital-risque institutionnel et développement local, Mémoire de DEA, Université Paris I, Panthéon, Sorbonne, p. 66-87. 